\title{
Actual vs. perceived talkativeness as determinants of judged leadership, popularity, and likeableness
}

\author{
DAVID J. STANG and JOHN A. CASTELLANETA \\ Queens College of the City University of New York, Flushing, New York 11962 \\ and

\begin{abstract}
GEORGE CONSTANTINIDIS and CARLOS R. FORTUNO
New York University, New York, New York 10003
\end{abstract}

\begin{abstract}
Although talkativeness seems to have an important influence on social perception, there is little evidence on the best way to measure talkativeness. Nineteen small discussion groups met on four occasions, and members simultaneously ranked and rated each other for talkativeness, leadership, popularity, and likeableness using a new rank-rate scale. Observers of the groups tallied emitted and received utterance frequency. Although there was adequate inter-observer agreement, these "objective" measures of talkativeness were only weakly related to the various peer judgments, while peer-judged talkativeness was closely and positively related to these variables. Subjective judgments appear to be more useful predictors of various social perceptions than objective tallies of emitted utterance frequency.
\end{abstract}

Over the years, researchers have observed that talkativeness seems to have an important influence on social perception. Numerous studies (reviewed in Stang, 1973) have reported positive correlations between talkativeness and peer judgments of leadership, popularity, and likeableness. Recently, it has been found (Stang, 1973) that experimentally manipulated talkativeness can affect these peer judgments, and that there seem to be extensive stereotypes of talkative and quiet people which agree with these laboratory findings (Stang \& Russell, Note 1).

One general question which has not been examined in this literature concerns how talkativeness should be assessed. When observers assess talkativeness and count utterance frequency, they face the problem of deciding whether to include in their tallies brief utterances such as attempted interruptions and interjections. Further, a simple count of utterance frequency may not be closely related to total speaking time, since some group members may speak on only a few occasions, but then speak at great length. In short, we may question the interrater reliability and the validity of "objective" mea-

We would like to thank the other researchers involved in this study: Shelley Adatto, Jay Bertin, Barnett Bezme, Joseph Faranda, Judy Kelly, Mary Jo McDermott, Deborah Meringolo, Michael Namer, Susan Novrotsky, Thomas Reap, William Riggio, Janice Smith, and Joseph Tuchinsky. We would also like to thank Donald Pascoe of the City University Mutual Benefit Instructional Network for running the video equipment. Requests for reprints should be sent to David Stang, American Psychological Association, 1200 17th Street N.W., Washington, D.C. 20036. sures of talkativeness. We may further question whether "objective" frequency of talking or peer-judged talkativeness is a better predictor of peer-judged leadership, popularity, and likeableness. These questions are explored in the present study.

Obtaining peer rankings or ratings has often posed problems for cohesive groups in previous research. Ranking members on some evaluative dimension is inevitably unpleasant, since the technique prohibits the rater from indicating a high opinion of all group members. Further, ranking forces equal-interval scale values onto members, when, in fact, the distances between some members are usually perceived as much greater than the distances between others. On the other hand, rating using 7-point scales is unsatisfactory; while members can indicate their absolute feelings toward other members, their relative feelings are less easily expressed, and 7-point scales may not discriminate very well. Consequently, in the study reported below, we employed a new combination of these scales: Each group member simultaneously ranked and rated all other group members by writing their names along the same vertical 5-in line-a "rank-rate scale"-to indicate his feelings about the other's absolute standings relative to the end points of the scale, and relative standings in relationship to other group members. The rank-rate scale thus enables a group member to evaluate all other members highly, if desired, while requiring (since ties were not permitted) a discrimination between these members on the rated dimension. Ratings of a group member on a scale were determined as the physical distance from one end of the scale to that member's name. 


\section{METHOD}

Sixteen observers, undergraduate students taking an advanced course in research methods at Queens College, were trained to tally utterance frequency in small group observations by collectively observing a small group with a one-way mirror and video monitors. Discussion among the observers while making these observations was geared toward maximizing inter-rater agreement. Nineteen small discussion groups of four to six members were formed from 73 female and 27 male students enrolled in three sections of an introductory social psychology course at Queens College; each group met for $1 / 2 \mathrm{~h}$ during each of 4 consecutive weeks. Participants in the small groups were volunteers, but were given course credit for participation. All group members were informed they would be observed and had brief opportunities to interact with the observer. Fourteen of the groups met in a room equipped with video monitors and a oneway mirror. The remaining five groups met in rooms lacking monitors and mirrors, and were observed by observers seated outside the group's circle.

During each group session, one or (usually) two observers recorded a tally in a matrix each time a group member spoke, the row indicating speaker, the column indicating person spoken to. The diagonal in this matrix was used to record utterances directed to the group as a whole, rather than toward a specific group member.

After each group session, participants were supplied with a booklet containing five rank-rate scales, one scale per page. Scales were anchored with "most (X) possible" and "least (X) possible," where (X) stood for leading in the group session, leadership ability in general, popularity in the group session, talkativeness in the session, and likeableness in the session. A cover page provided instructions and an example of how to use the scales.

\section{RESULTS}

Each group member's mean rating on each of the five peer-judged dimensions was found for each session. The observers tally of each member's emitted utterance frequency (number of times speaking) and received utterance frequency (number of times spoken to) was found and divided by the group size -1 , to adjust for the modest negative relationship $(r=-.10, p=.07)$ between these variables. Correlations across group members, sessions, and groups, are reported in Table 1.

The inter-observer agreement on emitted utterance frequency was .78 , while agreement on received utterance frequency was .72. Against these modest reliabilities may be seen the relationship between talkativeness as judged by other group members and talkativeness as judged by the trained observer: $r=.31$.

Examination of Table 1 suggests a closer relationship between judged talkativeness and the other judged vari-

Table 1

\begin{tabular}{lcccccc}
\hline & UR & JT & JL & JLA & JP & JLK \\
\hline Utterances emitted (UE) & .48 & .29 & .31 & .29 & .22 & .10 \\
Utterances received (UR) & & .16 & .17 & .19 & .12 & .07 \\
Judged talkativeness (JT) & & & .89 & .89 & .84 & .72 \\
Judged leading (JL) & & & & .90 & .84 & .74 \\
Judged leadership ability (JLA) & & & & & .85 & .77 \\
Judged popularity (JP) & & & & & & .90 \\
Judged likeableness (JLK) & & & & & &
\end{tabular}

Note-For $n=241$, if $r>.14, p<.05 ;$ if $r>.18, p<.01$. ables than between objectively measured talkativeness and the other judged variables. A principal-components factor analysis was performed on the five judged variables, emitted utterance frequency, and received utterance frequency. Two strong factors emerged: a peer-judgment factor (64\% of the total variance), with high loadings only from the five peer judgments, and an observer judgment factor ( $20 \%$ of the total variance), with high loadings only from the two observed judgments, emitted utterance frequency, and received utterance frequency. The factor analysis thus confirms the suggestion from Table 1 that peer judgments of talkativeness may be better predictors of other peer judgments than are observed judgments.

Further confirmation of this was obtained from multiple regression analyses using emitted utterance frequency, received utterance frequency, and peer-judged talkativeness as predictors of the other four peer judgments. In all four equations, peer-judged talkativeness made strong positive significant contributions ( $F$ ratios for this term ranging from 270 to 838 !). In none of the equations did received utterance frequency make a significant contribution. Only in predicting judged liking did emitted utterance frequency matter, and here the beta was -.13 , while the beta for peer-judged talkativeness was .76. In short, when peer judgments of talkativeness were available, the weak positive relationship between emitted utterance frequency or received utterance frequency and other peer-judged variables washed out.

These results would be explained if there were linear relationships between utterance frequency and received utterance frequency, and among the peer judgments, and nonlinear relationships between emitted utterance frequency and peer judgments. While there is some evidence (e.g., Matlin \& Stang, 1975; Stang \& Matlin, 1975 ) that judged frequency is an increasing concave downward function of actual frequency, that is not the case here: All relationships were essentially linear, with only a suggestion of leveling off at the higher emitted utterance frequencies. In fact, none of the tests for deviation from linearity for the relationship between each of the five judged variables and emitted or received utterance frequency were significant.

\section{DISCUSSION}

One explanation of these findings would suggest a halo effect: People nondiscriminatingly gave high ratings on all variables, including judgments of talkativeness, to those group members they most liked. This explanation is weakened, however, by other research (Matlin \& Stang, Note 2; Stang \& Russell, Note 1) which suggests that neither talkativeness nor quietness is a socially desirable quality. For instance, in these studies, about as many people judge themselves or their friends as above average in talkativeness as judged self or friends as below average in talkativeness.

Another possible explanation of these findings is that, insofar as talkativeness affects social perception, counts of emitted utterance frequency are not very efficient means of assessing this variable. Subsequent research may lead to better means of 
objectively measuring talkativeness, means which better predict judged talkativeness and its correlates.

Other research might more closely examine the properties of the rank-rate scale used here. Group members readily understood how to use the scale, and offered no objection to using it. Intuitively, the rank-rate scale seems potentially more valid and reliable than either ratings or rankings along, since it obtains information of both sorts. Such assessments are currently in progress.

\section{REFERENCE NOTES}

1. Stang, D. J. \& Russell, V. Stereotypes and self-descriptions of talkative and quiet people. Unpublished manuscript, Queens College, 1976.

2. Matlin, M. W. \& Stang, D. J. The Pollyanna principle:
Affect and evaluation in language, memory, and cognition. Book in Preparation, 1976.

\section{REFERENCES}

Matlin, M. W. \& Stang, D. J. Some determinants of word frequency estimates. Perceptual and Motor Skills, 1975, 40. 923-929.

StANG, D. J. Effect of interaction rate on ratings of leadership and liking. Journal of Personality and Social Psychology, 1973, 27, 405-408.

Stang, D. J. \& Matlin, M. W. Effect of meaningfulness and instructions on frequency estimation. Psychological Reports, 1975, 36, 164.

(Received for publication March 21, 1976.) 